\title{
Rupture rates after laparoscopic myomectomy using single stitches in only one layer
}

\author{
A. Kavallaris - C. Panayotidis $\cdot$ N. Chalvatzas $\cdot$ \\ C. Banz $\cdot$ K. Diedrich $\cdot$ A. Hornemann
}

Received: 12 February 2010 /Accepted: 22 March 2010/Published online: 20 April 2010

(C) Springer-Verlag 2010

\begin{abstract}
Myomectomy is a common laparoscopic procedure and is often used in patients with infertility, bleeding disorders and other symptoms caused by leiomyomas. We present a case series report based on a retrospective audit conducted from January 2001 up to December 2006 in our department. From 451 patients laparoscopically operated for leiomyomas, we identified only 59 patients operated due to infertility reasons. We report the post-operative rates of pregnancy and mode of delivery after a median followup of 40 months post-operatively. Laparoscopic technique and obstetrical outcome is discussed with recent literature review. The average number of removed fibroids was 2 . The mean weight of the leiomyomas was $94.3 \mathrm{~g}$. The cavum uteri was opened in eight patients. Overall, 42 out of 59 women delivered 51 live newborn babies, yielding a post-operative success rate of $71 \%$. The miscarriage rate post-operatively was 8 out of 60 pregnancies $(13 \%)$. In patients with leiomyomas identified as infertility cofactor, laparoscopic management is a convincing therapeutic approach. In our experience, conception rate was $71 \%$, and complications during pregnancy were limited to $4 \%$ of the patients. Risk of uterine rupture during labour was present in $4 \%$ of the cases, implying that mode of delivery should always be discussed with the patient.
\end{abstract}

\footnotetext{
A. Kavallaris $(\bowtie) \cdot$ N. Chalvatzas $\cdot$ C. Banz $\cdot$ K. Diedrich •

A. Hornemann

Department of Obstetrics and Gynecology,

Medical University of Luebeck,

Ratzeburger Allee 160,

23538 Luebeck, Germany

e-mail: andreas.kavallaris@uk-sh.de

C. Panayotidis

Department of Obstetrics and Gynecology,

Southend University Hospital,

Essex, UK
}

Keywords Laparoscopy · Myomectomy · Single stitch · Fibroids

\section{Background}

The most common uterine tumours are leiomyomas [1]. The prevalence in fertile women is described to be between $20 \%$ and $40 \%[2,3]$ and depends on their age and ethnic origin. Although most of the leiomyomas are asymptomatic, variable symptoms due to their location and size have been described from dysmenorrhoea, menorrhagia, to chronic pelvic pains and in some cases infertility.

Medical management of symptomatic leiomyomas have been vastly described such as the use of GnRHAnalogues [2,3], and during their use pregnancy is not an option. Prolonged use increases significantly the risk of osteoporosis [4], and therefore, long-term use is not recommended. The use of medical treatment reduces the volume of the fibroids, and therefore less blood loss was described [2, 3, 6]. Conservative management can be appropriate in certain cases when there is no significant patient morbidity in comparison with the risks and complication from a myomectomy.

Infertility is related with the presence of leiomyomas; therefore, conservative surgical management is needed to preserve fertility function post myomectomy. For conservative surgical management, different techniques have been proposed such as myomectomy, myomembolization, focused ultrasound technique [5] and cryomyolysis [6]. Myomectomies can be performed via open approach (laparotomy) or endoscopic approach (hysteroscopy or laparoscopically) [7, 8]. Fertility function post myomectomy is difficult to assess, and very heterogeneous data have been published regarding pregnancy rates [7-10]. In 
case of successful pregnancy, there are reports showing increased complication rates of uterine rupture during labour especially after laparoscopic myomectomy $[9,10]$. Other studies could not confirm these findings $[11,12]$. In view of the small number of case series regarding laparoscopic myomectomy and pregnancy rates, we have undertaken a retrospective audit of 59 consecutive cases for the treatment of infertility where the laparoscopic myomectomy was performed using single knots in one layer closure in order to assess the post-operative pregnancy rates.

\section{Methods}

We have identified retrospectively all laparoscopic myomectomies that have been submitted to our hospital from January 2001 to December 2006. Four hundred and fifty-one patients had benefited from laparoscopic myomectomy. From this group of patients, only 65 were nulliparous and had been presented with infertility problems. All operations were performed in our university hospital by three experienced laparoscopic surgeons. During this period, Laparoscopic myomectomy was performed in 451 patients. Basic principles of our techniques were respecting as much as possible the uterine anatomy (avoidance of any unnecessary trauma) without excessive bipolar coagulation and reconstitution of the uterine anatomy using single-layer closure technique with interrupted single stitches of absorbable 0 Vicryl sutures. Regarding the laparoscopic access, at least three trocars were used for the operative myomectomy, and all suture knots were made intracorporeally.

The leiomyomas were laparoscopically retrieved after the use of morcellator. In every patient, a blue dye test was done at the end of the operation in order to assess if the uterine cavity was opened or not and if the fallopian tubes were patent.

The audit was limited to the period for which detailed data were available from the existent patient files. All patients with infertility had median follow-up after laparoscopic myomectomy of 40 months (range, 60-94 months). All patients were followed up using a questionnaire.

We have sent to all 65 patients a questionnaire asking about their pregnancy outcome after the operation in our department.

\section{Findings}

From the 65 patients with infertility, a follow-up was possible for only 59 patients.

At the time of the myomectomy, the mean age of the patients was 34.5 (range, 25-37 years). The mean weight of the removed leiomyomas was $94.3 \mathrm{~g}(0.5 \mathrm{~g}-800 \mathrm{~g})$.
The average number of leiomyomas per patient was 2 (range, 4-13 fibroids). The median operative time was 68 min (range, 45-212 min). Intramural leiomyomas were found in all patients, and during the myomectomy the endometrial cavity was disrupted only in 8 of the 59 patients. All procedures were successfully managed laparoscopically with no conversion to laparotomy. The conception rate after the myomectomy was $78 \%$ in total. Forty-six patients of the 59 got pregnant post-operatively with a total of 60 pregnancies ( 35 got pregnant one time, eight patients got pregnant two times, and three patients got pregnant three times). The miscarriage rate post-operatively was 8 out of 60 pregnancies $(13 \%)$. In one patient, an ectopic pregnancy was diagnosed. Overall, 42 out of 59 women delivered 51 live newborn babies, yielding a postoperative success rate of $71 \%$.

A vaginal delivery was achieved in 32 deliveries (62.7\%). In ten $(19.5 \%)$ deliveries, elective Caesarian sections were performed. This decision was taken by the responsible obstetrician as precaution from possible uterine rupture during labour. In five deliveries, an elective Caesarian section was performed due to maternal request $(9.8 \%)$. In the remaining four cases, an emergency Caesarean section was necessary. Two crash Caesarean sections were performed due to rupture of the uterus (4\%) and two emergency Caesarean sections due to abnormal cardiotogographic monitoring (4\%).

\section{Discussion}

The overall incidence of uterine rupture during delivery is about $0.05 \%$ [13]. Thirteen percent of these uterine ruptures occur in the unscarred uterus. An increased risk of rupture or dehiscence of the uterus during pregnancy and labour is seen in patients after any surgical procedure involving the uterus. The risk of uterine rupture after a Caesarean section is described to be up to $1.5 \%$ [14-17]. A history of multiple Caesarean deliveries seems not to be associated with an increased rate of uterine rupture in women attempting vaginal birth compared with those with a single prior operation. All 59 patients were nulliparous and have been diagnosed as infertile because of leiomyomas. During the laparoscopic procedure, single-layer closure technique was used with interrupted single stitches of absorbable 0 Vicryl. There were no intra- or post-operative complications. There were no tubal obstructions. In all 59 patients, the fallopian tubes were patent, and in eight (13.5\%) patients, the uterine cavity was opened.

The incidence of scar rupture following open myomectomy was described to be as high as 5.3\% [16]. Our investigation showed a comparable result for the laparoscopic approach. In our patients, an emergency Caesarean section was necessary for uterine rupture in $4 \%$ (two cases). 
We believe that in the two cases of uterine rupture during labour, neither the location nor the fibroid volume represented an important factor in predicting the rupture; rather, they have in common the opening of the uterine cavity. Yet, in both cases, the location of the leiomyomas and the scar was am fundus uteri, and the rupture during labour was on the low uterine segment, far away from the scar of the myomectomy. We suppose that the rupture is more a multifactorial result rather than a direct consequence of the myomectomy. However, the most important finding of our study is a post-operative live birth rate of $71 \%$ and vaginal delivery of $62.7 \%$ in a median follow-up period of 40 months. Comparing our results with similar studies [7, $11,12]$, we have the highest live birth rate of $71 \%$ and the lowest Caesarean section rate of $37.3 \%$.

\section{Conclusion}

In infertile patients with fibroids, the laparoscopic management seems to be an adequate procedure that does not decrease the conception rate post-operatively. We have observed a risk for uterine rupture during labour (4\%); therefore, the mode of delivery should be discussed carefully with these patients.

Declaration of interest The authors report no conflicts of interest. The authors alone are responsible for the content and writing of the paper.

\section{References}

1. Vollenhoven B (1998) Introduction: the epidemiology of uterine leiomyomas. Baillieres Clin Obstet Gynaecol 12:169-176

2. Wallach EE, Vlahos NF (2004) Uterine myomas: an overview of development, clinical features, and management. Obstet Gynecol 104:393-406

3. Stewart EA (2001) Uterine fibroids. Lancet 357:293-298
4. Lefebvre G, Vilos G, Allaire C, Jeffrey J, Arneja J, Birch C, Fortier M, Wagner MS (2003) The management of uterine leiomyomas. J Obstet Gynaecol Can 25:396-418, quiz 419-322

5. Tempany CM, Stewart EA, McDannold N, Quade BJ, Jolesz FA, Hynynen K (2003) MR imaging-guided focused ultrasound surgery of uterine leiomyomas: a feasibility study. Radiology 226:897-905

6. Zreik TG, Rutherford TJ, Palter SF, Troiano RN, Williams E, Brown JM, Olive DL (1998) Cryomyolysis, a new procedure for the conservative treatment of uterine fibroids. J Am Assoc Gynecol Laparosc 5:33-38

7. Ribeiro SC, Reich H, Rosenberg J, Guglielminetti E, Vidali A (1999) Laparoscopic myomectomy and pregnancy outcome in infertile patients. Fertil Steril 71:571-574

8. Marret H, Chevillot M, Giraudeau B (2004) A retrospective multicentre study comparing myomectomy by laparoscopy and laparotomy in current surgical practice. What are the best patient selection criteria? Eur J Obstet Gynecol Reprod Biol 117:82-86

9. Grande N, Catalano GF, Ferrari S, Marana R (2005) Spontaneous uterine rupture at 27 weeks of pregnancy after laparoscopic myomectomy. J Minim Invasive Gynecol 12:301

10. Lieng M, Istre O, Langebrekke A (2004) Uterine rupture after laparoscopic myomectomy. J Am Assoc Gynecol Laparosc 11:9293

11. Paul PG, Koshy AK, Thomas T (2006) Pregnancy outcomes following laparoscopic myomectomy and single-layer myometrial closure. Hum Reprod 21:3278-3281

12. Seinera P, Farina C, Todros T (2000) Laparoscopic myomectomy and subsequent pregnancy: results in 54 patients. Hum Reprod 15:1993-1996

13. Zwart JJ, Richters JM, Ory F, de Vries JI, Bloemenkamp KW, van Roosmalen J (2009) Uterine rupture in The Netherlands: a nationwide population-based cohort study. Bjog 116:1069-1078, discussion 1078-1080

14. Farmer RM, Kirschbaum T, Potter D, Strong TH, Medearis AL (1991) Uterine rupture during trial of labor after previous cesarean section. Am J Obstet Gynecol 165:996-1001

15. Landon MB, Spong CY, Thom E, Hauth JC, Bloom SL, Varner MW, Moawad AH, Caritis SN, Harper M, Wapner RJ et al (2006) Risk of uterine rupture with a trial of labor in women with multiple and single prior cesarean delivery. Obstet Gynecol 108:12-20

16. Roopnarinesingh S, Suratsingh J, Roopnarinesingh A (1985) The obstetric outcome of patients with previous myomectomy or hysterotomy. West Indian Med J 34:59-62

17. Hurst BS, Matthews ML, Marshburn PB (2005) Laparoscopic myomectomy for symptomatic uterine myomas. Fertil Steril 83:123 